\title{
Robust routing and timetabling in complex railway stations
}

\author{
Sofie Burggraeve ${ }^{a}$ \\ sofie.burggraeve@kuleuven.be, tel: +3216372023 \\ Pieter Vansteenwegen ${ }^{a}$ \\ pieter.vansteenwegen@kuleuven.be \\ ${ }^{a}$ KU Leuven Mobility Research Centre - CIB \\ Celestijnenlaan 300 BOX 2422, 3001 Leuven \\ BELGIUM
}




\title{
Robust routing and timetabling in complex railway stations
}

\begin{abstract}
In nearly saturated station areas the limited capacity is one of the main reasons of delay propagation. Spreading the trains well in time and space in these areas has a big impact on the passenger robustness, i.e. the total travel time in practice of all passengers in the railway network in case of frequently occurring small delays. We focus on improving the performance in the bottleneck of the network in order to improve the performance of the whole railway network. This paper proposes a method that builds from scratch a routing plan and a cyclic timetable that optimizes the infrastructure occupation and the passenger robustness. An integer linear routing model assigns, without considering a timetable, every train to a route such that the maximal node usage is minimized and that the number of times that each node is used, is quadratically penalized. Thereafter, a mixed integer linear timetabling model assigns to each train the blocking times at which the nodes on its route, assigned by the routing model, are reserved and released. Different from other approaches is that we focus on the occupation of the railway infrastructure before constructing the timetable. The approach is validated on the complex railway station area of Brussels (Belgium). Our routing plan and timetable from scratch improve the passenger robustness up to $11 \%$ compared to a reference timetable and routing plan composed by the Belgian railway infrastructure manager Infrabel and by up to $2 \%$ compared to a reference timetable and routing plan from literature.
\end{abstract}

Keywords: Passenger robustness; Railway routing; Railway timetabling; (mixed) integer linear program 


\section{Introduction}

Railway bottlenecks are characterized by dense train traffic and a complex infrastructure lay-out. It goes without saying that both characteristics make the planning of a bottleneck complicated, bút crucial for the performance of the whole network. Therefore it is useful to focus on the planning of a bottleneck before planning the rest of the network (Goldratt, 1986). Thereafter the planning can normally be extended and made feasible for the whole network outside this bottleneck without many changes, since typically much less constraints are present outside the bottleneck. In the first place, we want to construct a conflict-free schedule for the bottleneck, which means that no two trains block the same infrastructure at the same time (Caimi, 2009). Obviously, railway passengers want both short and reliable travel times. Hence, in the second place our objective is to optimize the passenger robustness, which means minimizing the total travel time of all passengers in practice in case of frequently occurring small delays (Dewilde et al., 2011). Unfortunately, direct implementation of this objective function is computationally highly demanding, as real travel times of all passengers and propagation of delays have to be calculated. Therefore we indirectly strive for passenger robustness by looking for an optimal spreading of the trains in time and space. We restrict our research to timetabling and routing, which are situated on the tactical level of railway planning. However, also line planning, on the structural level, and real-time interventions, on the operational level, have an impact on the travel times of railway passengers in practice and thus on the passenger robustness of the railway system. The timetable and routing plan construction are only designed to mitigate the effect of frequently occurring small delays on the passenger travel times. The impact of large disturbances is not considered during the construction of the timetable or routing plan. Nevertheless, we are convinced that this optimal spreading can also be useful in case of larger disturbances. To summarize, the focus of this research is on making a conflict-free and passenger robust timetable and a routing plan from scratch to transport passengers optimally in, through and out of a railway bottleneck. If a number of stations close to each other serve a high proportion of the passengers, it typically becomes a bottleneck in the railway system. We will refer to this set of stations as $a$ station area. A station area can be divided in platform areas (one platform area in each station) and grid zones in between these platform areas.

The main contributions of this paper are

- A routing model and a timetabling model to construct a passenger robust and conflict-free routing plan and timetable from scratch.

- A validation of this routing model and timetabling model on a realistic, large and complex railway bottleneck, namely Brussels (Belgium).

- A routing plan and timetable which significantly improve the passenger robustness of reference routing plans and timetables from practice and from literature. 
- Additive and alternative constraints to speed up routing and timetabling models and to include transfers, re-usage, splitting and coupling of trains into these models.

The proposed routing and timetabling model can be used in sequence as we illustrate in this paper. However, they can also be used independently from each other. First, in Section 2, we introduce some definitions and describe how our method is related to the state of the art in timetabling and routing for nearly saturated railway station areas. We also point out the novelties and differences of our approach. Secondly, our methodology is explained in detail. The optimization models are introduced and illustrated on a small example case study in Section 3 . In Section 4 , the input of the case study on the railway bottleneck in Brussels is presented together with some alternative and additional constraints to model the splitting, coupling and re-usage of trains and to speed up the models. The performance of the presented method is discussed in Section 5 . The paper is concluded and ideas for future research are presented in Section 6 .

\section{State of the art}

A railway network can be represented by a graph where switches, platforms and network border points are nodes and the tracks between these nodes are the links. From now on we will refer to switches, platforms and border nodes as nodes. A route in the railway network is a sequence of succeeding nodes and links. A routing plan is an assignment of trains to routes. It should be noted that for a station area the line planning already fixed where the trains enter and leave the area. The route planning then determines which nodes and links the trains use inside the station area. A microscopic timetable is an assignment of blocking times (reservation and release times) to the

links and nodes on the trains' routes. For a macroscopic timetable, a routing plan is not necessarily assigned yet. A macroscopic timetable is an assignment, for each train, of arrival and departure times in each station on the train's line. The construction and optimization of a routing plan and a timetable are closely interwoven. The order in which both problems are solved, determines the complexity, the restrictions and the objectives of both problems. In case the timetabling problem is solved first, only a macroscopic timetable can be constructed. The routing problem, which is solved thereafter, is then constrained by the macroscopic timetable. Moreover, the routing plan fixes at the same time a microscopic timetable. Thus, in this case, the routing plan is only feasible if it incurs a conflict-free microscopic timetable. Not every macroscopic timetable, however, does assure this existence of a feasible routing plan (Sels, 2016b). Thus, only after solving the routing problem, a statement can be made about the conflict-freeness of the schedule. In case the routing problem is solved first, the routing plan is not constrained by the timetable. Once the routing plan is known, not only a macroscopic timetable, but immediately a microscopic timetable can be designed. In this case the timetable is constrained by the routing plan. But also here, a conflict-free timetable does 
not exist for every routing plan. Only after solving the timetabling problem, a statement can be made about the conflict-freeness of the schedule. Thus, both a routing plan and a timetable are necessary to judge the conflict-freeness of the schedule.

For large networks with many stations but relatively simple infrastructure lay-out or relatively sparse traffic, it is advantageous to first construct a macroscopic timetable and only thereafter consider the routes of the trains. In a railway bottleneck, by contrast, it could be advantageous to immediately look at the microscopic infrastructure level for the construction of an optimal routing plan and timetable. This could lead to a more efficient use of the available infrastructure, as we will illustrate for our case study.

We now give a literature overview to situate our research. We divide the related approaches into three categories: approaches to construct a routing plan, approaches to construct a timetable and approaches that solve the integrated problem. For each approach we indicate which input is required, so which order of the routing and the timetabling problems this approach assumes.

The approaches that solve the integrated problem mostly consist of a combination of a routing approach that can be categorized in the first class and a timetabling approach, that can be classified in the second class, and optionally a feedback loop between both approaches. To avoid repetition and overlap, we will not discuss these routing and timetabling approaches separately, but only in the part on integrated approaches. Although our approach also tackles both problems succeedingly, we will compare our solution approach for the routing problem to the approaches in the first class and our solution approach for the timetable to the approaches in the second class. In that second class, we also introduce the necessary terminology to describe a microscopic timetable.

\subsection{Routing problem}

In literature, research that only focuses on the routing problem is sparse and it almost always starts from a macroscopic timetable. So in that case the routing problem is restricted by already scheduled arrival and departure times or bounds. Apart from finding a conflict-free assignment of trains to the routes, these approaches optimize a given objective function while adhering (or only slightly modifying) the given macroscopic timetable. Examples of optimization criteria are maximizing the number of trains that can be routed, minimizing the shunting movements, maximizing the preferences of trains for certain platforms or routes, minimizing average travel time, minimizing energy consumption, maximizing user satisfaction, robustness etc. (e.g. Zwaneveld et al., 1996, 2001, Caimi et al. 2011; Sels, 2014, Sun et al., 2014). Sun et al. (2014) gives a short literature overview on routing problems in their paper.

Our routing approach is different, since in our research the routing problem precedes the timetabling problem. We are not restricted by the timetable, i.e. no timings nor orders are fixed, so we are free to spread (all) the trains over the available infrastructure. We want that as little trains as possible 
interact with each other. We achieve this by smartly minimizing the node usage over the whole network. Since longer routes and detour routes use more nodes, these will also be avoided by our objective function. Another application of our routing model could be to estimate and judge the spreading of a routing plan constructed for a given timetable. Our routing model could be used to calculate a reference unrestricted spreading of the trains in space.

\subsection{Timetabling problem}

Existing timetabling models commonly focus only on the macroscopic level. Most models solving this problem are based on the periodic event scheduling problem (PESP) (e.g. Serafini and Ukovich, 1989, Kroon et al., 2009, Cacchiani et al., 2012, Liebchen et al., 2007; Schmidt and Schöbel, 2015). In the PESP, arrival and departure times of trains in stations are events. Related events are linked to each other by constraints that put an upper bound and/or lower bound on the time duration between these events. Examples of related events are arrival and departure times of the same train, arrival and departure times of trains that provide a transfer, arrival and departure times of trains using the same platform, etc.

However, there also exist approaches that focuses on microscopic timetabling. While the PESP is suitable to model the microscopic timetabling problem, no results hereof are published. Furthermore, during our preliminary experiments on the microscopic level, we found out that it is more efficient to use auxiliary linear reservation and release times to construct our microscopic timetable than to use the PESP approach. Before we discuss the existing approaches, we first introduce the terminology necessary to describe a microscopic timetable. Apart from nodes and links a railway network also contains signals to safely guide the trains through the network. A section is defined to be a part of the railway network between two signals. A section can contain (part of) links and zero up to several nodes. A train reserves a section and the nodes in this section on the instant that this section is allocated exclusively to this train. Then the section is also blocked for other trains. This time instant is referred to as the reservation time. The blocking time is the total time a section is allocated exclusively to a train movement and therefore blocked for other trains. The train releases a section and all infrastructure elements in this section at the end of the blocking time. This time instant is referred to as the release time. The construction of a microscopic timetable consists of the assignment of reservation and release times to the sections on the trains' routes. The assignment of reservation and release times has to be conflict-free, so no two trains are planned to block the same infrastructure element at the same time (Caimi, 2009). Buffer times and supplements are two important characteristics of a timetable. A buffer time between two trains in an infrastructure element is the time between the blocking times of these two trains on that infrastructure element. A supplement is an amount of time that is added to the minimum necessary running or dwell time of a single train. Buffer times are useful for avoiding delay propagation and do not affect the travel 
times (run and/or dwell times) of the trains. Supplements are useful for absorbing delays but do increase the planned travel times of the trains and the passengers. Remark that buffer times only affect the planned passenger travel time in case of transfers.

To the best of our knowledge, only integrated approaches design a microscopic timetable from scratch. However, there exist approaches that start from an existing microscopic timetable and only focus on improving the robustness of this initial timetable. They achieve this by only making (relatively) small changes to the initial timetable while sticking to the initial routing plan. Andersson et al. (2015) and Kroon et al. (2008) improve the location of buffer times and/or the distribution of supplements in the timetable. Fischetti et al. (2009) alters the timetable in order to improve the robustness by using a set of delay scenarios. The advantage is that these small changes can have a relatively large impact on the performance of the railway system and that these changes can easily be implemented in practice without affecting the passengers too much. The disadvantage is that the final result is still highly dependent on the initial timetable.

In this paper, we construct a microscopic timetable from scratch, but we first consider the construction of the routing plan. Our objective is to create a passenger robust microscopic timetable. We achieve this by maximizing the buffer times between the trains. Since we start from scratch and first build a routing plan, our final result is not dependent on an initial timetable. We can thoroughly work out a globally optimal solution without being constrained to small changes and without any bias towards solutions that are similar to the initial timetable.

\subsection{Integrated routing and timetabling problem}

As is the case for timetabling approaches, there exist integrated approaches that start from an initial schedule and approaches that start from the ground up. The method proposed in this paper consists of a separate routing model and timetabling model without feedback loop between the models. So this method is not obviously categorized as an integrated method. However, the routing model constructs a routing plan with the design of a timetable by the timetabling model in mind: the intensity with which a node is used determines the maximum possible buffer time in that node and thus the vulnerability of that node for propagating delays. Spreading out the node usage as much as possible enables and facilitates the construction of a passenger robust timetable in the next step.

A minority of the integrated approaches start from an initial schedule. Examples with a large positive impact on the performance of the railway system are Dewilde et al. (2013, 2014); Burggraeve et al. (2015). These works start from an initial routing plan and timetable and iterate between a routing module, a timetabling module and a platforming module in order to improve the passenger robustness of the schedule. We maintain the same objective in this research, but we start from the ground up and obtain better results. 
Integrated methods that start from the ground up mostly consist of a feedback loop between the microscopic and the macroscopic level, while in this paper all scheduling is performed on the microscopic scale. In these other approaches arrival and departure times are assigned on the macroscopic level. Thereafter a routing plan is developed and the microscopic timetable which is created thereby is tested on its conflict-freeness, e.g. by simulation or an analytic approach. Research that belongs to this category is for example Sels (2016b). This work is also constructed for and validated on the Belgian railway network and optimizes passenger robustness. However, while we only focus on the bottleneck, Sels et al. (2016a) and Sels (2016b) focus on a larger part of the network. They get overall good results but not when specifically looking at the bottleneck. Another difference is that Sels et al. (2016a) and Sels (2016b) explicitly constrain the buffer times between different trains on a common node by imposing headway times, while we do not constrain these buffer times but maximize them in the objective function of the optimization model. This is possible since we immediately work on the microscopic scale. Other examples in this category are Zwaneveld et al. 1996, 2001); Kroon et al. (2009) and Schlechte et al. (2011). Also the research of (Lamorgese et al. 2016 belongs to this category. Interestingly, they propose an exact model to solve the routing and the timetabling problem at the same time, but they use a micro-macro heuristic to actually calculate the routing plan and timetable. Furthermore, the number of route alternatives in their case study is small and they do not take any form of robustness into account. By contrast (Caimi et al. 2011) proposes a multi-level framework for generating train schedules in highly utilized networks. Their approach works fast, but they encounter that the level of detail used to create the macroscopic schedule highly affects the result. With the problems they encounter, it seems hard to find an appropriate macroscopic network lay-out that simplifies the complex microscopic infrastructure layout considered in this paper. Furthermore, also here, robustness is not considered. More advanced micro-macro approaches also detect places sensitive for delay propagation, for example based on an infrastructure occupation rate higher than the recommended threshold by UIC $(2013)$ in (Bešinović et al., 2016; Goverde et al., 2016, Bešinović et al., 2017). These works manage delay propagation by including or removing supplements to dwell and running times of the appropriate trains, but this can also cause new conflicts. These detected conflicts are excluded by going back to the macroscopic level and assigning new arrival and departure times while dealing with this information. The final timetable is a conflict-free, stable and robust timetable. The main difference with our work is the infrastructure lay-out and train density for which the method is designed. Another difference is that Bešinović et al. (2016); Goverde et al. (2016) use heuristic approaches, while we make use of exact optimization models. The advantage is that with our approach the routing plan and the timetable can be optimally designed for the bottleneck. However, due to the complex infrastructure lay-out and the train density, our computation time is large. 


\section{Methodology}

\subsection{Routing model}

The routing model assigns every train to a route. A route is defined as a sequence of succeeding nodes and links. We focus on the nodes because they uniquely determine the route of a train through the network. Moreover, two trains can only be in conflict if they share at least one node. It is possible that trains don't share a (complete) section but that they only share one node (as a cross point of two sections). Furthermore, the infrastructure occupation of a railway system can be expressed by the usage of the nodes in the network. We make no assumptions about the timetable as the timetable will only be determined after the routing plan. However, the routing plan is constructed such that a robust timetable can be built. The routing model assigns every train to a route such that every node is used as little as possible. Consequentially, the trains are spread in space and the infrastructure of the station area is optimally used. Moreover, the idea is that the less train traffic there is on a node, the easier it will be to pull trains apart in time during timetabling in order to construct a passenger robust timetable.

\subsubsection{Objective function}

In order to achieve that every node is used as little as possible, we combine two aspects. We explicitly minimize the maximum use of any node. We say that a node is used $x$ times if there are $x$ trains whose route contains this node. Furthermore, we minimize the sum of the squares of the usages of all nodes. We minimize the sum of the squares of the usages, instead of for example the sum of the usages, to penalize an increase in a node utilization rate harder the higher the utilization rate of the node already is. This second objective gives the incentive to further decrease the individual node usages. Intuitively, slalom routes pass more nodes and thus will negatively affect our objective function. The two minimization problems are integrated into one problem by giving the minimization of the maximum node usage a much higher weight $(\mathcal{H})$. The magnitude of $\mathcal{H}$ depends on the problem size. We now present and explain the optimization model.

\subsubsection{Parameters}

$T=\left\{t_{1}, t_{2}, \cdots, t_{|T|}\right\} \quad=$ set of trains with $|T|$ the number of trains.

$W \quad=\left\{w_{1}, w_{2}, \cdots, w_{|W|}\right\}=$ set of nodes with $|W|$ the number of nodes.

$R=\left\{r_{1}, r_{2}, \cdots, r_{|R|}\right\} \quad=\quad$ set of routes with $|R|$ the number of routes.

$R_{t} \subset R=$ set of routes that train $t$ can be assigned to (based on its incoming and outgoing line in the station area). 
$l_{r, w}=1$ if route $r \in R$ contains node $w \in W$ and

$=0$ otherwise.

$\mathcal{H}=$ the high weight used to enforce the domination of the minimax criterion in the objective function of the routing model.

\subsubsection{Decision variables}

$$
\begin{array}{ll}
g_{w} \in \mathbb{Z}^{+} & =\text {number of times node } w \text { is used, } w \in W . \\
x_{t, r} & =1 \text { if train } t \in T \text { is assigned to route } r \in R_{t} \text { and } \\
& =0 \text { otherwise. } \\
z & =\text { maximum node usage overall. }
\end{array}
$$

\subsubsection{Model}

The combined weighted objective function of the minimization of the maximum node usage and the minimization of the sum of the squared node usages, can be represented by

$$
\min \quad \mathcal{H} \max _{w \in W} g_{w}+\sum_{w \in W} g_{w}^{2}
$$

Remark that both terms of this objective function are non-linear. The first part is a minimax objective and can easily be linearized by introducing decision variable $z$ and constraints (6). The second part is harder to linearize as it consists of a sum of squared, but bounded integers. In Burggraeve et al. 2015) a method is proposed. We compactly summarize this method and propose a partly different implementation which proved to be significantly better in computation time for our case study. Introduce $K|W|$ binary decision variables $b_{k, w}$, with $k \in\{1, \cdots, K\} \subset \mathbb{Z}^{+}, w \in W$ and with $K$ an upper bound on the maximum node usage. A train can pass a node at most once, so in any case $K \leq|T|$. The $b_{k, w}$ 's are defined as

$$
b_{k, w}=\left\{\begin{array}{lll}
1 & \text { if } & g_{w} \geq k \\
0 & \text { if } & g_{w} \leq k-1
\end{array}\right.
$$

If node $w$ is used at least $k$ times $\left(g_{w} \geq k\right)$, then $b_{k, w}=1$, else $b_{k, w}=0$. The sum of squared node usages is converted to a sum of linear sums by using the coefficients $c_{k}$ given in Table 1 for each node $w$ :

$$
\sum_{k=1}^{K} c_{k} b_{k, w}=\sum_{k=1}^{g_{w}} c_{k}=g_{w}^{2}
$$

where the first equality is true by definition of the $b_{k, w}$ 's. 
Table 1: Linearization of the quadratic terms

\begin{tabular}{ccc}
\hline & $c_{m}$ & $\sum_{k=1}^{m} c_{k}$ \\
\hline$m \in \mathbb{Z}^{+}$ & $2 m-1$ & $m^{2}$
\end{tabular}

In Burggraeve et al. (2015), the following constraints were proposed

$$
g_{w}-K b_{k, w} \leq k-1 \quad \forall k \in\{1, \cdots, K\}, \forall w \in W
$$

Instead of these constraints, we now propose constraints (7) to define the $b_{k, w}$ 's in this minimization problem. Both implementations give rise to the same optimal value. However, the number of constraints in (7) is only $|W|$ compared to $K|W|$ in (4). The computation times of both methods are compared in Section 5 . This results in the following integer linear routing model:

$$
\begin{array}{cll}
\min & \mathcal{H} z+\sum_{w \in W}\left(\sum_{k=1}^{K} c_{k} b_{k, w}\right) & \\
\text { s.t. } & g_{w} \leq z & \forall w \in W \\
g_{w}=\sum_{k=1}^{K} b_{k, w} & \forall w \in W \\
\sum_{r \in R_{t}} x_{t, r}=1 & \forall t \in T \\
\sum_{t \in T} \sum_{r \in R_{t}} l_{r, w} x_{t, r}=g_{w} & \forall w \in W \\
g_{w} \in \mathbb{Z}^{+} & \forall w \in W \\
z \in \mathbb{Z}^{+} & \\
x_{t, r} \in\{0,1\} & \forall t \in T, \forall r \in R_{t} \\
b_{k, w} \in\{0,1\} & \forall k \in\{1, \cdots, K\}, w \in W .
\end{array}
$$

Constraints (8) assure that each train is assigned to exactly one route that connects the incoming and outgoing line of that train in the network. Constraints (9) assure that $g_{w}$ equals the number of trains that will use node $w$. Constraints $(10)$ and $(12)$ assure that the number of times that a node is used is a positive integer and that the assignment of a train to a route is represented by the binary variable $x_{t, r}$. If the $b_{k, w}$ 's would only be defined as continuous variables in [0,1], instead of binary variables in (13), and $z$ as a continuous variable in $\mathbb{R}_{0}^{+}$, instead of a positive integer in (11), these variables would still be forced to be binary, resp. integral, due to the model construction.

\subsection{Timetabling model}

We propose a new mixed integer linear model to construct a cyclic timetable from scratch. The overall objective is to build a conflict-free and passenger robust timetable on microscopic scale. The 
timetabling model starts from a microscopic routing plan, for example the routing plan assigned by the routing model. The dwell and running times and the lengths of the blocking times of the sections on the train's route can be calculated beforehand based on the characteristics of the train, the section and the supplement distribution. These blocking time lengths are used to fix time durations that indicate the reservation and release times of the sections on the train's route relative to the entrance time of the train at the border node of its incoming line. These time durations are used as parameters in the timetabling model. The reservation and release times of nodes that are part of the same section are the same for one train.

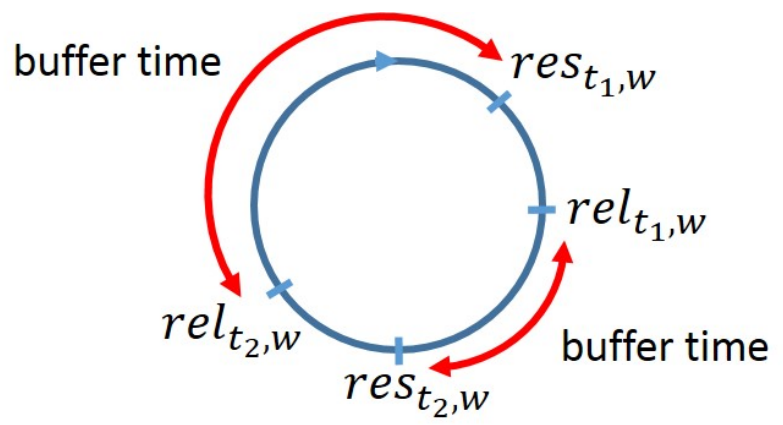

Figure 1: Train $t_{1}$ reserves and releases the node on time instants $\operatorname{res}_{t_{1}, w}$ and $\operatorname{rel}_{t_{1}, w}$. Train $t_{2}$ reserves and releases that same node on time instants $\operatorname{res}_{t_{2}, w}$ and $\mathrm{rel}_{t_{2}, w}$. A first buffer time is the one between rel $_{t_{1}, w}$ and res $_{t_{2}, w}$ and the second buffer time is the one between $\operatorname{rel}_{t_{2}, w}$ and $\operatorname{res}_{t_{1}, w}$.

\subsubsection{Objective function}

The objective function of the timetabling model is to maximize the buffer times between trains. The buffer times in a certain node between two trains (which trips are repeated every period) are presented on a cyclic time axis in Figure 1. No decisions on supplements are included in the model. In Section 5, we present the effect of different amounts of dwell time supplements on the performance of the timetable. Remark that the more supplements are added in the timetable, the less time and capacity is left for including buffer times between train pairs.

To optimize the buffer times between the trains, we again focus on two aspects. The approach is similar to that of the routing model. Primary, we maximize the minimum buffer time over all nodes, i.e. the smallest amount of time between the reserve and release times of trains on any node. Secondary, we maximize the sum of the minimum buffer times between every two trains having at least one node in common. This second optimization objective gives the incentive to further increase the buffer times between train pairs. We again combine these two objectives by summing them up and giving a high weight $(\widetilde{\mathcal{H}})$, depending on the problem size, to the minimum overall buffer time. 


\subsubsection{Parameters}

$$
\begin{aligned}
& r^{t} \quad=\text { the route that is assigned to train } t \in T \text { in the routing plan. } \\
& s_{r}\left(s_{r^{t}}\right) \quad=\text { the first node on route } r\left(r^{t}\right) \text {. } \\
& d_{t, r^{t}, w}^{\mathrm{res}}\left(d_{t, r^{t}, w}^{\mathrm{rel}}\right)=\text { the time duration for train } t \in T \text { to reserve (release) node } w \\
& \text { relatively to the entrance of train } t \text { in the network. } \\
& T_{w} \quad=\quad \text { the set of trains } t \text { for which } r^{t} \text { contains node } w \text {. } \\
& P \quad=\text { the period length of the cyclic timetable. } \\
& \widetilde{\mathcal{H}} \quad=\text { the high weight used to enforce the domination of the maximin criterion } \\
& \text { in the objective function of the timetabling model. }
\end{aligned}
$$

\subsubsection{Decision variables}

Let $\xi$ be the smallest integer such that all $d_{t, r^{t}, w}^{\text {res }}$ and $d_{t, r^{t}, w}^{\text {rel }}$ are smaller than $(\xi-1) P$.

$$
\begin{aligned}
& \operatorname{res}_{t, w}, \operatorname{rel}_{t, w} \in[0, P[\quad=\text { the moment at which train } t \in T \text { reserves, resp. releases } \\
& \text { node } w \text { in the cyclic timetable, } \\
& \forall t \in T, w \in r^{t} . \\
& \operatorname{res}_{t, w}^{\text {aux }}, \operatorname{rel}_{t, w}^{\text {aux }} \in[0, \xi P[\quad=\quad \text { the moment at which train } t \in T \text { reserves, resp. releases } \\
& \text { node } w \text { in case of a linear (instead of a cyclic) timetable, } \\
& \forall t \in T, w \in r^{t} . \\
& \operatorname{res}_{t, w}^{\mathrm{int}}, \operatorname{rel}_{t, w}^{\mathrm{int}} \in\{0,1, \cdots, \xi-1\}=h \text { if } \operatorname{res}_{t, w}^{\mathrm{aux}}, \operatorname{resp} . \operatorname{rel}_{t, w}^{\mathrm{aux}} \in[h P,(h+1) P[\text {, } \\
& \forall t \in T, w \in r^{t}, h \in\{0,1, \cdots, \xi-1\} \subset \mathbb{Z}^{+} . \\
& \text {resrel }_{t_{i}, t_{j}, w}^{\mathrm{int}} \in\{-\xi, \cdots, \xi\} \quad=h \text { if } \operatorname{res}_{t_{j}, w}^{\text {aux }}+h P-\operatorname{rel}_{t_{i}, w}^{\text {aux }} \in[0, P[\text {, } \\
& \forall t_{i}, t_{j} \in T: i<j, w \in r^{t_{i}} \cap r^{t_{j}}, h \in\{-\xi, \cdots, \xi\} \subset \mathbb{Z} \text {. } \\
& \operatorname{buf}_{t_{i}, t_{j}, w} \in\left[0, P\left[\quad=\text { the buffer time between the reservation time of train } t_{j}\right. \text {, }\right. \\
& \text { and the release time of train } t_{i} \text { in node } w \text {, } \\
& \forall t_{i}, t_{j} \in T: t_{i} \neq t_{j}, w \in r^{t_{i}} \cap r^{t_{j}} . \\
& \operatorname{minbuf}_{t_{i}, t_{j}} \in[0, P / 2] \quad=\text { the minimum buffer time between train } t_{i} \text { and } t_{j} \\
& =\min \left\{\operatorname{buf}_{t, t^{\prime}, w} \mid t, t^{\prime} \in\left\{t_{i}, t_{j}\right\}: t \neq t^{\prime}, w \in r^{t_{i}} \cap r^{t_{j}}\right\} \\
& \forall t_{i}, t_{j} \in T: t_{i} \neq t_{j}, r^{t_{i}} \cap r^{t_{j}} \neq \emptyset . \\
& \tilde{z} \in[0, P / 2] \quad=\text { the minimum buffer time overall. }
\end{aligned}
$$

\subsubsection{Model}

The maximization of the minimum buffer time over all nodes can be represented by:

$$
\max \min \left\{\operatorname{buf}_{t_{i}, t_{j}, w} \mid w \in W,\left(t_{i}, t_{j}\right) \in T_{w} \times T_{w}: t_{i} \neq t_{j}\right\}
$$


The maximization of the sum of the minimum buffer times between every two trains can be represented by:

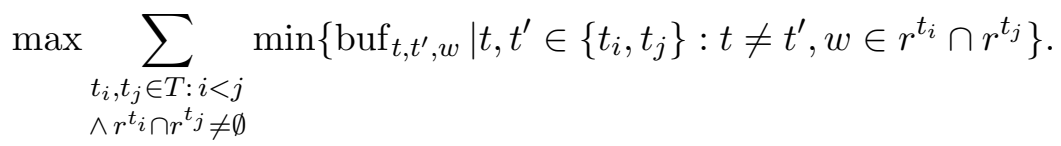

Both parts of the objective function are or can be reduced to maximin objectives. Objective function (16) together with constraints (17) (for the first objective part) and constraints (18) (for the second objective part) present a linearization of the combined weighted objective function. The timetabling model then becomes:

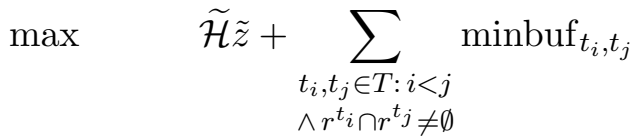

$$
\begin{aligned}
& \text { s.t. } \quad \tilde{z} \leq \operatorname{buf}_{t_{i}, t_{j}, w} \quad \forall w \in W, t_{i}, t_{j} \in T_{w}: t_{i} \neq t_{j} \\
& \operatorname{minbuf}_{t_{\min \{i, j\}}, t_{\max \{i, j\}}} \leq \operatorname{buf}_{t_{i}, t_{j}, w} \quad \forall t_{i}, t_{j} \in T: t_{i} \neq t_{j}, w \in r^{t_{i}} \cap r^{t_{j}} \\
& \operatorname{res}_{t_{j}, w}^{\text {aux }}-\operatorname{rel}_{t_{i}, w}^{\text {aux }} \geq-P \text { resrel }_{t_{i}, t_{j}, w}^{\text {int }} \quad \forall w \in W, \forall t_{i}, t_{j} \in T_{w}: i<j \\
& \operatorname{res}_{t_{i}, w}^{\mathrm{aux}}-\operatorname{rel}_{t_{j}, w}^{\mathrm{aux}} \geq-P\left(1-\operatorname{resrel}_{t_{i}, t_{j}, w}^{\mathrm{int}_{1}}\right) \quad \forall w \in W, \forall t_{i}, t_{j} \in T_{w}: i<j \\
& \operatorname{buf}_{t_{i}, t_{j}, w} \leq \operatorname{res}_{t_{j}, w}^{\text {aux }}-\operatorname{rel}_{t_{i}, w}^{\text {aux }}+P \operatorname{resrel}_{t_{i}, t_{j}, w}^{\text {int }} \quad \forall w \in W, \forall t_{i}, t_{j} \in T_{w}: i<j \\
& \operatorname{buf}_{t_{j}, t_{i}, w} \leq \operatorname{res}_{t_{i}, w}^{\text {aux }}-\operatorname{rel}_{t_{j}, w}^{\text {aux }}+P\left(1-\operatorname{resrel}_{t_{i}, t_{j}, w}^{\mathrm{int}}\right) \quad \forall w \in W, \forall t_{i}, t_{j} \in T_{w}: i<j \\
& \operatorname{res}_{t, w}^{\mathrm{aux}}-\operatorname{res}_{t, s_{r} t}=d_{t, r^{t}, w}^{\mathrm{res}} \quad \forall t \in T, \forall w \in r^{t} \\
& \operatorname{rel}_{t, w}^{\text {aux }}-\operatorname{res}_{t, s_{r} t}=d_{t, r^{t}, w}^{\mathrm{rel}} \quad \forall t \in T, \forall w \in r^{t} \\
& 0 \leq \operatorname{res}_{t, w}^{\text {aux }}-P \operatorname{res}_{t, w}^{\mathrm{int}}<P \quad \forall w \in W, \forall t \in T_{w} \\
& \operatorname{res}_{t, w}=\operatorname{res}_{t, w}^{\mathrm{aux}}-P \operatorname{res}_{t, w}^{\mathrm{int}} \quad \forall w \in W, \forall t \in T_{w} \\
& 0 \leq \operatorname{rel}_{t, w}^{\text {aux }}-P \operatorname{rel}_{t, w}^{\mathrm{int}}<P \quad \forall w \in W, \forall t \in T_{w} \\
& \operatorname{rel}_{t, w}=\operatorname{rel}_{t, w}^{\mathrm{aux}}-P \operatorname{rel}_{t, w}^{\mathrm{int}} \quad \forall w \in W, \forall t \in T_{w} \\
& \tilde{z} \in[0, P[ \\
& \operatorname{minbuf}_{t_{i}, t_{j}} \in[0, P / 2] \quad \forall t_{i}, t_{j} \in T: i<j \\
& \operatorname{buf}_{t_{i}, t_{j}, w} \in\left[0, P\left[\quad \forall w \in W, \forall t_{i}, t_{j} \in T_{w}: t_{i} \neq t_{j}\right.\right. \\
& \operatorname{res}_{t_{i}, w}, \operatorname{rel}_{t_{i}, w} \in\left[0, P\left[\quad \forall w \in W, \forall t_{i} \in T_{w}\right.\right. \\
& \operatorname{res}_{t_{i}, w}^{\text {aux }}, \operatorname{rel}_{t_{i}, w}^{\text {aux }} \in\left[0, \xi P\left[\quad \forall w \in W, \forall t_{i} \in T_{w}\right.\right. \\
& \operatorname{res}_{t_{i}, w}^{\mathrm{int}}, \operatorname{rel}_{t_{i}, w}^{\mathrm{int}} \in\{0, \cdots, \xi-1\} \subset \mathbb{Z} \quad \forall w \in W, \forall t_{i} \in T_{w} \\
& \operatorname{resrel}_{t_{i}, t_{j}, w}^{\mathrm{int}} \in\{-\xi, \cdots, \xi\} \subset \mathbb{Z} \quad \forall w \in W, \forall t_{i}, t_{j} \in T_{w}: i<j
\end{aligned}
$$


Constraints 190 - 20 assure that the blocking times of two trains that share a node do not overlap. These constraints need some extra explanation. In a linear (non-cyclic) timetable, blocking times of a train pair $\left(t_{i}, t_{j}\right)$ for a certain node $w$ do not overlap if the reservation time of one of the two trains is later than the release time of the other of the two trains, i.e. $\operatorname{res}_{t_{i}, w}-\operatorname{rel}_{t_{j}, w} \geq 0$ or $\operatorname{res}_{t_{j}, w}-\operatorname{rel}_{t_{i}, w} \geq 0$. However, in a cyclic timetable, this condition can be fulfilled for the auxiliary reservation and release times or for the reservation and release times itself while the blocking times do overlap. An example of each is provided in Table 2, considering that the period length $P$ equals 60 minutes. A negative result in line 1 and 2 or 3 and 4 for the last two columns indicates an overlap of blocking times. Constraints 120 exclude these cases and force that the blocking times of different trains for a shared node cannot overlap. The auxiliary variable resrel $_{t_{i}, t_{j}, w}^{\mathrm{int}}(i<j)$ is a measure of how many times the period length has to be added to the auxiliary release time of the train with index $j$ such that the buffer time in node $w$, buf $_{t_{i}, t_{j}, w}$, can be calculated as the auxiliary release time of train $j$ plus resrel $\mathrm{l}_{t_{i}, t_{j}, w}^{\mathrm{int}}$ times the period length minus the auxiliary reservation time of train $i$. The buffer time between two trains in a shared node is defined by constraints 21)-(22). Constraints (23)-24 link the reservation time of the start node to the reservation and release time of the other nodes on the route of a train, based on the duration parameters. Constraints 25)-28 link the auxiliary reservation and release times to the (cyclic) reservation and release times.

Table 2: Example of (non)-straightforward overlapping blocking times.

\begin{tabular}{c|c|cc|c|cc} 
train & $\mathrm{i}$ & $\left(\mathrm{res}_{t_{i}, w}^{\mathrm{aux}}, \mathrm{rel}_{t_{i}, w}^{\mathrm{aux}}\right)$ & $\left(\mathrm{res}_{t_{i}, w}, \mathrm{rel}_{t_{i}, w}\right)$ & $(i, j)$ & $\operatorname{res}_{t_{i}, w}^{\mathrm{aux}}-\mathrm{rel}_{t_{j}, w}^{\mathrm{aux}}$ & $\mathrm{res}_{t_{i}, w}-\mathrm{rel}_{t_{j}, w}$ \\
\hline$t_{1}$ & 1 & $(2,5)$ & $(2,5)$ & $(1,2)$ & $2-66=-64$ & $2-6=-4$ \\
$t_{2}$ & 2 & $(63,66)$ & $(3,6)$ & $(2,1)$ & $63-5=58$ & $3-5=-2$ \\
$t_{3}$ & 3 & $(58,61)$ & $(58,1)$ & $(3,4)$ & $58-62=-4$ & $58-2=56$ \\
$t_{4}$ & 4 & $(59,62)$ & $(59,2)$ & $(4,3)$ & $59-61=-2$ & $59-1=58$
\end{tabular}

\subsection{Small example case study}

We demonstrate our approach on the fictive and schematic network in Figure 2, The bold circles represent switches or border nodes, the rectangles are platforms and the flags are signals which demarcate the sections on the network. Signals indicated in blue (black) are for trains driving from left (right) to right (left). Instead of calculating detailed blocking times, we here use simple calculations to fix the time duration parameters. The numbers next to the arrows present the time in minutes necessary to get from one end of the corresponding link to the other end. We suppose that a train needs 0.2 minutes to get its tail out of a section once its head left that section. The necessary stop time at the station is two minutes. We consider six trains on our network for which the incoming and outgoing lines are fixed in advance. Each train has up to four possible routes. The output of the routing model and the timetabling model is visualized in Figure 3 . The maximum 


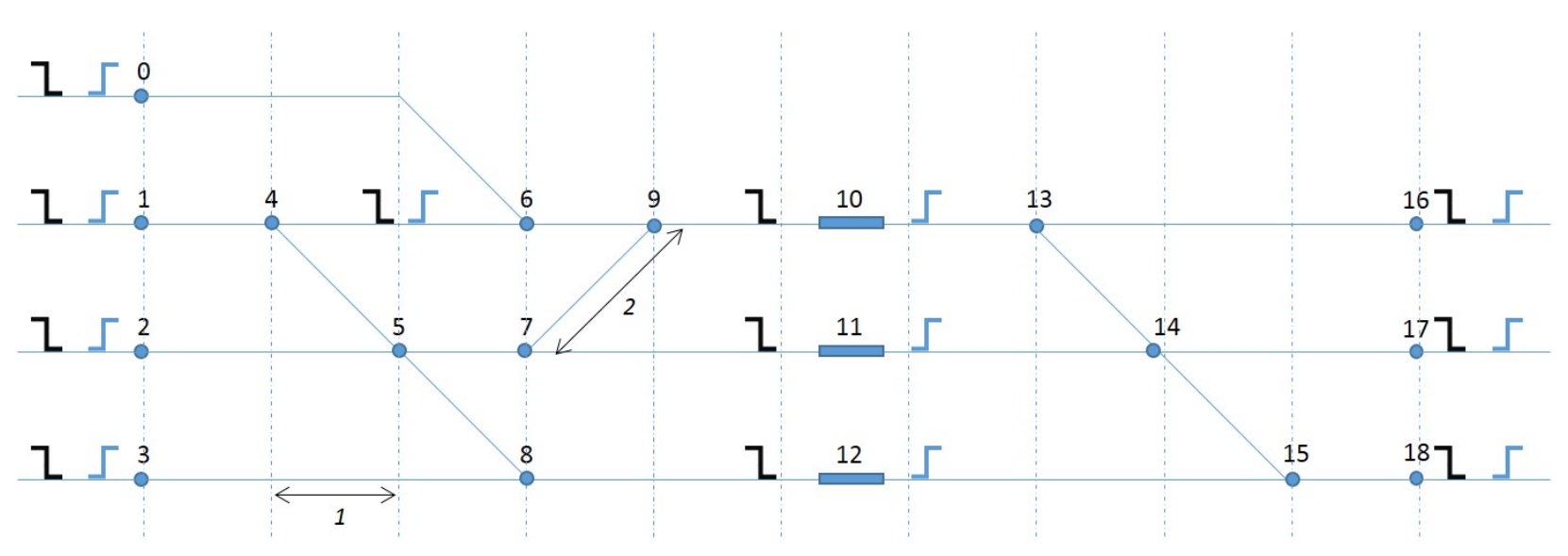

Figure 2: Network of the small example case study

number of times that a node is used is three (node 5), which already implies that the minimum buffer time over all nodes can never be higher than 20, considering that the period length equals 60 minutes. The sum of the squared node usages is 75 . Table 3 presents the reservation and release times of each train in all the nodes on its route. The nodes are put together per section. Table 4 shows the minimum buffer times between each train pair that shares at least one node. The minimum buffer time overall is 12.13 minutes, which occurs between the reservation and release times of train 0,3 and 4 in node 5 . Note that node 5 is blocked for 23.6 minutes by these three trains, so the buffer times are equally spread in this node. The sum of the minimum buffer times between every train pair that shares at least one node is 102.79 minutes.
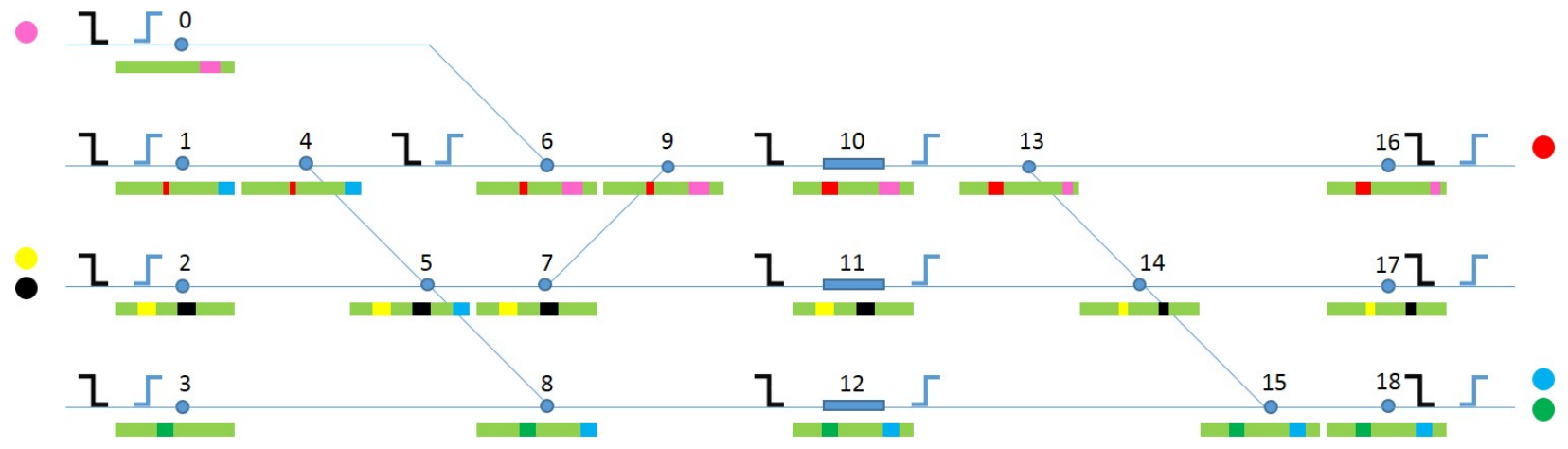

Figure 3: For each of the six trains a colored ball indicates the border node of its incoming line. The small rectangles below the nodes present a time axis of one hour indicating when the nodes are free and blocked. Parts indicated in light green are buffer times and the other colors indicate the blocking times of the different trains. 
Table 3: The reservation and release times for each train in all the nodes on its route.

\begin{tabular}{cccrr} 
Train $t$ & color & nodes $w$ & res $_{t, w}$ & rel $_{t, w}$ \\
\hline 0 & $\bullet$ & $18 ; 15 ; 12$ & 45.80 & 53.00 \\
& & $8 ; 5 ; 4 ; 1$ & 52.80 & 0.00 \\
1 & $\bullet$ & $18 ; 15 ; 12$ & 15.80 & 23.00 \\
& & $8 ; 3$ & 22.80 & 30.00 \\
2 & $\bullet$ & $16 ; 13 ; 10$ & 15.30 & 22.50 \\
& & $9 ; 6$ & 22.30 & 25.50 \\
& & $4 ; 1$ & 25.30 & 27.50 \\
3 & $\bullet$ & $2 ; 5 ; 7 ; 11$ & 12.13 & 20.33 \\
& & $14 ; 17$ & 20.13 & 24.33 \\
4 & $\bullet$ & $2 ; 5 ; 7 ; 11$ & 32.47 & 40.67 \\
& & $14 ; 17$ & 40.47 & 44.67 \\
5 & $\bullet$ & $0 ; 6 ; 9 ; 10$ & 43.80 & 53.00 \\
& & $13 ; 16$ & 52.80 & 57.00 \\
\hline
\end{tabular}

Table 4: The minimum overall buffer time is $12.13 \mathrm{~min}-$ utes. The sum of the minimum buffer times between each pair that has at least one node in common is 102.79 minutes.

\begin{tabular}{cc} 
Train pair & $\begin{array}{c}\text { minimum } \\
\text { buffer time }\end{array}$ \\
\hline $0-1$ & $22.80 \mathrm{~min}$ \\
$0-2$ & $25.30 \mathrm{~min}$ \\
$0-3$ & $12.13 \mathrm{~min}$ \\
$0-4$ & $12.13 \mathrm{~min}$ \\
$2-5$ & $18.30 \mathrm{~min}$ \\
$3-4$ & $12.13 \mathrm{~min}$ \\
\hline
\end{tabular}

\section{Case study}

In order to validate the proposed models, we perform a case study on the railway station area of Brussels, which contains the top three busiest stations of Belgium 11 Our network also includes the beginning of the open tracks, the outer grids, and the entrances to the shunt yards. The core of this area is presented in Figure 4 and a schematic overview of the entire station area is presented in Figure $55^{2}$

Considering every border point, switch, i.e. point where two tracks cross, and platform as a node in the network, this leads up to 481 nodes. Brussels' station area contains six platform areas with 22 (Brussels South), 6 (Brussels Kapel), 6 (Brussels Central), 6 (Brussels Congres), 12 (Brussels North) and 12 (Schaarbeek) platforms. We consider 85 trains passing through this network during the morning peak hour between $7 \mathrm{am}$ and $8 \mathrm{am}$. We first describe the line planning that is used as input for the routing model. Thereafter, we describe the blocking times that are used as input for the timetabling model. Both the line planning and the data for the blocking time calculation correspond to data used by Dewilde et al. (2013, 2014) and are based on data from the Belgian railway infrastructure manager Infrabel. Next, we discuss some alternative and additional constraints which can be included into the models in order to (i) speed up the models, (ii) model the re-usage, splitting and coupling of trains and (iii) model transfers. We close this section by shortly describing the simulation tool that we use to measure the performance of different routing plans and timetables.

\footnotetext{
${ }^{1}$ Source: https://pvmagazine.nl/brussel-noord-nu-het-drukste-station-van-belgie/, consulted in July 2016

${ }^{2}$ Source: http://www.infrabel.be/sites/default/files/documents/ns_c-01-map-net-1045901_1.pdf, consulted in September 2014 .
} 


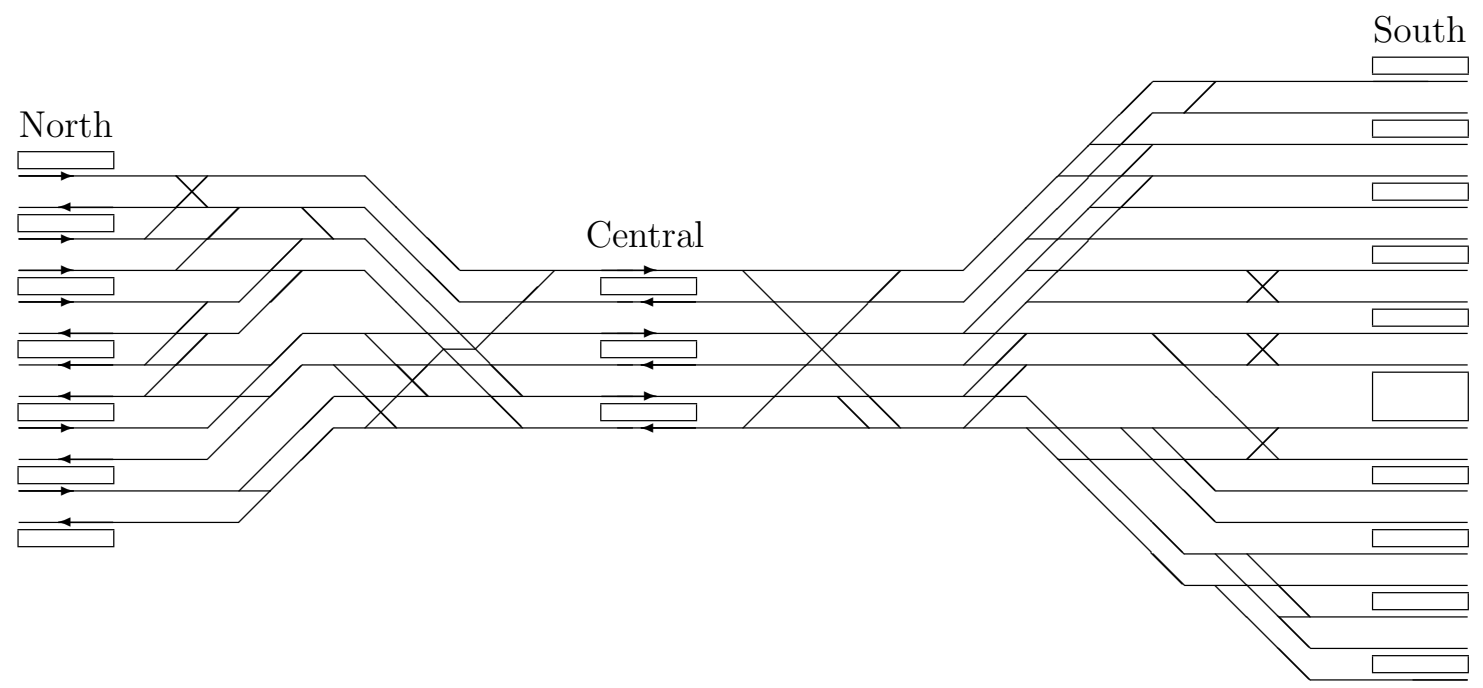

Figure 4: The core of the dense railway area of Brussels.

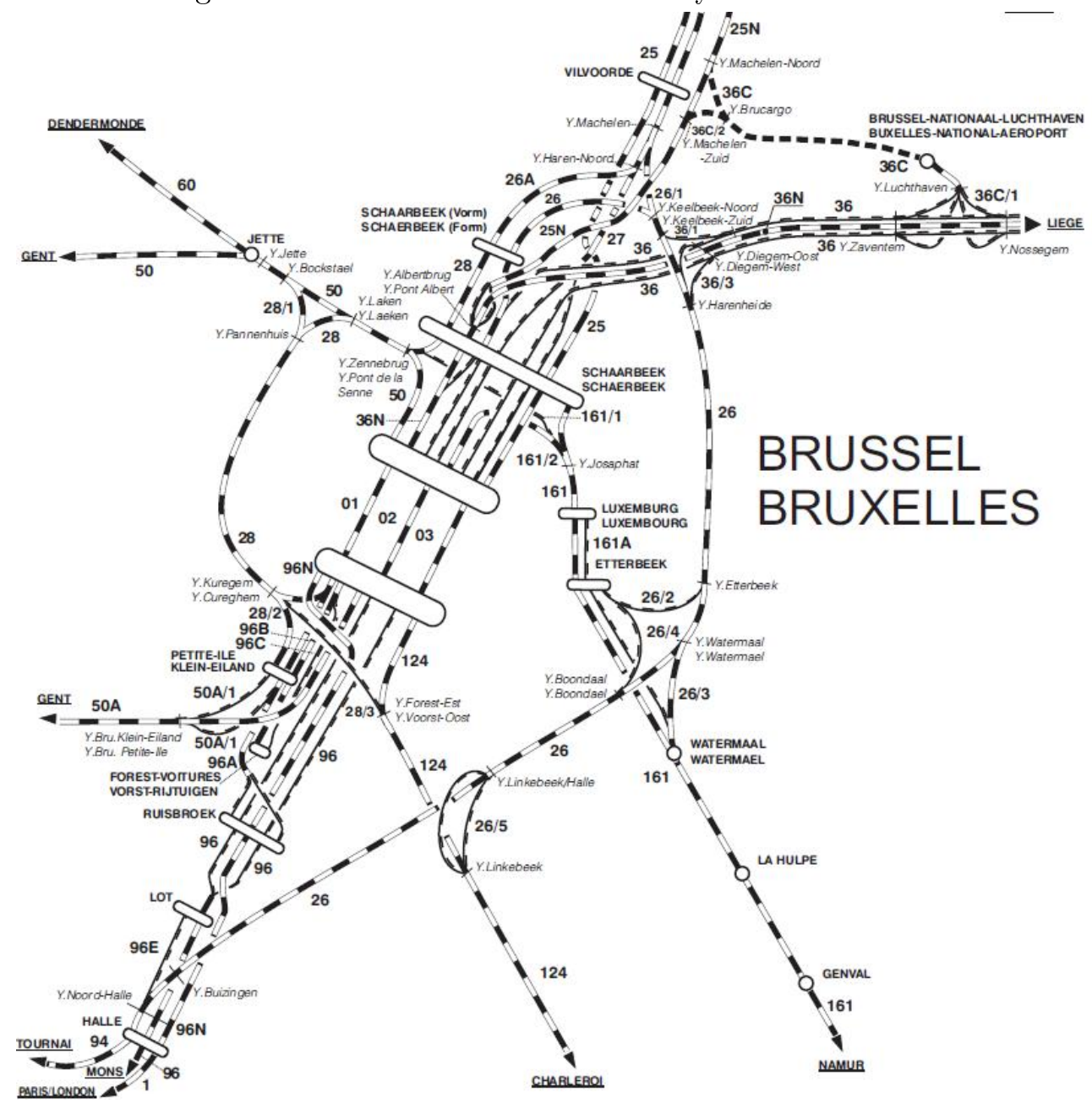

Figure 5: A schematic overview of the entire station area of Brussels. The tracks indicated by 01, 02 and 03 are a simplification of the core of Brussels dense railway area presented in Figure 4 . 


\subsection{Line planning}

In the line planning, there are 42 trains driving in the direction from South to North coming from six different incoming lines and leaving the network via eight different outgoing lines. Furthermore, there are 43 trains driving in the direction from North to South coming from seven different incoming lines and leaving the network via seven different outgoing lines. There are 73 trains traversing the whole network (from border node to border node). The other 12 trains start or end their route in a platform area. There is one train that splits into two trains in the platform area of Brussels South. There are also three trains that end their route in the platform area of Brussels South and are reused for a second trip starting from this platform area. All trains dwell in Brussels South, 76 in Brussels Central, 76 in Brussels North, 23 in Brussels Schaarbeek, ten in Brussels Congres and two in Brussels Kapel.

\subsection{Blocking times}

We here describe the data used in the case study to calculate the lengths of the blocking times, which is necessary to fix the time duration parameters $d_{t, r^{t}, w}^{\text {res }}$ and $d_{t, r^{t}, w}^{\text {rel }}$. Note that our timetabling model can be applied to any (part of a) network by appropriately assigning values to the duration

parameters $d_{t, r^{t}, w}^{\mathrm{res}}$ and $d_{t, r^{t}, w}^{\mathrm{rel}}$. This makes our timetabling model generally applicable; the only limit is the size and complexity of the network considered. The blocking time defined by Pachl (2008) consists of the time for setting up, watching the signal, approaching the section, traversing and clearing the section and releasing the signal and depends on the type of the train, on the route that the train is assigned to and the specific section on this route. Note that the release time of a section and the reserve time of the succeeding section are not equal due to the safety margins and since the tail of the train is still in the previous section when the head enters the next section. We approximate the time to traverse the section by dividing the length of the section by the speed of the train in that section and the time to leave that section. If the train enters a section in which the train stops at a platform, the minimum necessary dwell time for boarding and alighting of passengers (one up to five minutes) is added together with some additional time for braking to standstill and to accelerate back to the speed limit (up to 0.9 minutes). In consultation with Infrabel, we do not consider the possibility that a train needs multiple sections to brake because of the moderate train speeds in Brussel's station area. The amounts of additional time are based on data from Infrabel and depend on the type of the train, the direction of the train and the station of the stop. By definition all nodes that are part of the same section are reserved and released at the same moment. Only in case the section contains a platform, the nodes before the platform are already released from the moment the train has arrived on the platform. This design decision is also used by Infrabel in complex station areas. In order to compare the performance of our timetable with a reference timetable from Infrabel and from Dewilde et al. (2013), we added an amount of dwell time supplements equal to the amount 
of supplements present in their timetable. These dwell time supplements are added on top of the minimum necessary dwell time, each time a train stops on a platform. Furthermore, we lengthen the blocking time of the first section of a train with one minute, by advancing the reservation time, to account for the approach time to the considered restricted network. If a train leaves the network in one of the platform areas, the blocking time of the platform is lengthened with four extra minutes to account for the time that the train needs to free that platform. The time to clear the section is approximated with a fixed amount of time, independent of the train and section. These assumptions correspond to data from Infrabel. Since the available data does not explicitly contain values for the time for setting up, watching the signal, approaching the section and releasing the signal, we assume that these times are included in the available data. Due to this uncertainty in the data, we cannot assure conflict-freeness at the microscopic level for our case study. However, we can assure that the same data and principles to calculate the lengths of the blocking times are used by the Belgian railway infrastructure manager Infrabel and Dewilde et al. (2013) when planning complex station areas, so it makes sense to make a comparison of the resulting routing plans and timetables.

\subsection{Modeling improvements for routing and timetabling models}

\subsubsection{Flow conservation constraints to speed up the routing model}

The total number of routes starting at a border point or platform and ending at a border point or platform amounts 28970248 for Brussels' station area. As input, the routing model needs for each train the set of routes that connects the border node or platform of its incoming and outgoing line. For each of these (train, route)-combinations a variable has to be included in the model, which has to be considered in constraints $(9)$ for each node on that route. To decrease this computational effort, we divide the network in four parts to construct the routes. These parts are determined based on the most important platform areas. We call a route that is restricted to one part of the network a partial route. The four parts are indicated in Table 5, together with their number of partial routes in each direction. This division in partial routes significantly reduces the terms in constraints (9), which made it computationally possible to include all the routes in the routing model in a reasonable amount of time. However, the consecutive setting of partial routes for each

Table 5: The number of partial routes in each part of the network in two opposite directions (South to North and North to South).

\begin{tabular}{|c|c|c|c|}
\hline Network parts & & South to North & North to South \\
\hline Border (South) & - Brussels South & 1282 & 1824 \\
\hline Brussels South & - Brussels Central & 188 & 189 \\
\hline Brussels Central & - Brussels North & 27 & 28 \\
\hline Brussels North & - Border (North) & 913 & 802 \\
\hline
\end{tabular}


train needs to be included into the routing model. But, including this decision into the model not only reduced the model construction time, but also significantly reduced the computation time up to one hundredth. Constraints (8) are replaced by constraints (36)-(38):

$$
\begin{array}{lll}
\sum_{r \in \mathcal{R}_{\vec{t}, \mathrm{dep}, s_{r} t}} x_{t, r}= & 1 & \forall t \in T \\
\sum_{r \in \mathcal{R}_{\vec{t}, \mathrm{arr}, e_{r} t}} x_{t, r}= & 1 & \forall t \in T \\
\sum_{r \in \mathcal{R}_{\vec{t}, \mathrm{arr}, w}} x_{t, r} & =\sum_{r^{\prime} \in \mathcal{R}_{\vec{t}, \mathrm{dep}, w}} x_{t, r^{\prime}} \quad \forall t \in T, \forall w \in W \backslash\left\{s_{r^{t}}, e_{r^{t}}\right\},
\end{array}
$$

where $s_{r^{t}}$ and $e_{r^{t}}$ are respectively the border node of the incoming and outgoing line of train $t$ in

the network, $\mathcal{R}_{\vec{t}, \mathrm{dep}, w}\left(\mathcal{R}_{\vec{t}, \text { arr }, w}\right)$ contains all partial routes that depart from (arrive in) node $w$ in the direction of train $t$, indicated with $\vec{t}$. These constraints are flow conservation constraints for each train. They assure that (i) there is exactly one partial route assigned starting at the border node of the incoming line of the train (in the correct direction), constraints (36), (ii) there is exactly one partial route assigned arriving in the border node of the outgoing line of the train (in the correct direction), (37), (iii) in the intermediate parts of the network, partial routes are assigned such that one feasible, i.e. connected, route is built for each train, constraints (38). If a train enters the network in platform area $\mathcal{P}$ (and not in a border node of the network) and the platform is not decided beforehand, then we include for train $t$ as many binary variables as platforms $p$ there are in that platform area: $\operatorname{bin}_{p, t} \in\{0,1\}, \forall p \in \mathcal{P}$. We replace constraint (36) of train $t$ by the following constraints:

$$
\begin{aligned}
\sum_{p \in \mathcal{P}} \operatorname{bin}_{p, t} & =1 \\
\sum_{r \in \mathcal{R}_{\vec{t}, \mathrm{dep}, p}} x_{t, r} & =\operatorname{bin}_{p, t} \quad \forall p \in \mathcal{P} .
\end{aligned}
$$

So the train has to depart from exactly one platform in platform area $\mathcal{P}, \sqrt{39}$, , and has to be assigned to exactly one route that starts from that platform (and to none of the routes starting from the other platforms), (40). For trains which terminate their route in a platform area without a platform being fixed beforehand, analogous constraints are included.

\subsubsection{Constraints to speed up the timetabling model}

Based on the output of the routing model and the calculated blocking time lengths, we can calculate the total time that each node will be blocked in one period of the cyclic timetable before the timetable is actually constructed. We use this information to put an upper bound on the minimum buffer time 
in the timetabling model: the minimum buffer time will always be smaller than the maximum time that a node is blocked divided by the number of trains that pass in that node.

Secondly, note that two timetables that only differ by adding a fixed number to each arrival and departure time are in essence the same. We remove this symmetry from the model by fixing the reservation time of the first section of the train with the smallest index to zero.

\subsubsection{Splitting, coupling and re-usage of trains in routing and timetabling models}

In case a train is planned to split into two parts in a platform area, we schedule the first part as the original train, the second part is scheduled as a new train that starts its route from the platform where the splitting occurs. Each of these two trains has another outgoing line from then on. In case of coupling, the first train arriving at the platform is scheduled to finish its trajectory at the coupling platform and the second train arriving at the coupling platform continues as the coupled train from then on. In case a train is planned to be reused for a next line after it finishes a first line, both lines use the same physical train. However, we schedule the second trip of this train as a new train. This new train starts its route from the platform where the original train ended its route. For the splitting of trains and for the re-usage of a train, the new train needs to start from the same platform as where the original train is split, in case of splitting, or where the original train ended its first trip, in case of a re-usage. Therefore we add the next constraints in the routing model for every splitting and re-usage:

$$
\operatorname{bin}_{p, t^{\prime}}=\sum_{r \in \mathcal{R}_{\vec{t}, \text { arr }, p}} x_{t, r} \quad \forall p \in \mathcal{P}
$$

where $t$ is the original train and $t^{\prime}$ is the new train after splitting or re-usage and $\mathcal{P}$ is the platform area where the splitting or re-usage occurs. We use the $\operatorname{bin}_{p, t^{\prime}}$ variable, because it is not known before optimization on which platform the new train will start its trip through the network. For the coupling of trains the two trains have to arrive on the same platform. This can also be modeled by constraints (41) with $t$ the first train that arrives and $t^{\prime}$ the second train that arrives and $t^{\prime}$ is assumed to continue as the coupled train. Corresponding to the data of Infrabel, the time that is scheduled between the release time of the platform of both trains that take part in a splitting is three minutes. In the timetabling model, this can be caught in the following constraint:

$$
\operatorname{rel}_{t^{\prime}, p}=\operatorname{rel}_{t, p}+3
$$

where $p$ is the platform in $\mathcal{P}$ where the splitting takes place, which is already assigned by the routing model, $t$ is the original train in the splitting and $t^{\prime}$ is the new train arisen in the splitting. There is no prescribed time interval between the arrival and departure of the two trains that take part 
in a re-usage. To assure that passengers on the first train do not have to wait too long before the coupling takes place and the coupled train leaves the platform, constraint 42 can also be used

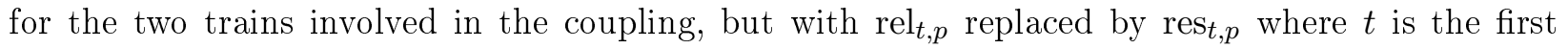
train arriving and $t^{\prime}$ is the second train arriving. Furthermore, the 'three' in constraint 42 must be replaced by an appropriate value. Since there are no trains coupling in our case study, we have no data on the coupling time. To prohibit that other trains are planned on the platform while the train that will be reused or coupled has arrived on the platform, but not yet left the platform, we include the following constraints in the timetabling model:

$$
0 \leq\left(\operatorname{res}_{t^{\prime \prime}, p}^{\mathrm{aux}}-\operatorname{rel}_{t, p}^{\mathrm{aux}}+P \operatorname{resre}_{t, t^{\prime \prime}, p}^{\mathrm{int}}\right)-\left(\operatorname{res}_{t^{\prime}, p}^{\mathrm{aux}}-\operatorname{rel}_{t, p}^{\mathrm{aux}}+P \operatorname{resrel}_{t, t^{\prime}, p}^{\mathrm{int}}\right) \leq P,
$$

where $t$ is the first train in the re-usage or coupling, $t^{\prime}$ is the second train in the re-usage or coupling and $t^{\prime \prime}$ is a third train that is planned to use the same platform on its route. These constraints assure that the reservation time of the second train of the re-usage is closer to the release time of the first train of this re-usage than the reservation time of any other train that makes use of this platform. Remark that this constraint is only valid if the index of train $t$ is smaller than that of train $t^{\prime}$ and $t^{\prime \prime}$, since the value and the sign of resrel $l_{t, t^{\prime}, p}^{\mathrm{int}}\left(\right.$ resrel $\left._{t, t^{\prime \prime}, p}^{\mathrm{int}}\right)$ depends on the order of the indices of train $t$ and $t^{\prime}\left(t\right.$ and $\left.t^{\prime \prime}\right)$. These constraints are changed accordingly for the other cases, i.e. index of train $t$ bigger than index of train $t^{\prime}$ but smaller than that of train $t^{\prime \prime}$, etc.

\subsubsection{Passenger transfers in routing and timetabling models}

To facilitate passenger transfers, the feeder and the connecting train could be planned on specific platforms in the routing model, for example adjacent platforms. Let $p$ be a platform in the platform area where the transfer is planned. Define $\mathcal{V}_{p}$ as the set of platforms for the connecting train which assure a fluent transfer in case the feeder train would arrive on platform $p$. This fluent transfer is then assured by adding the following constraint for each $p$ in the platform area where the transfer takes place

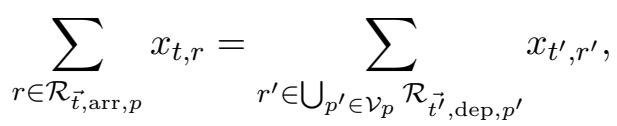

where $t$ is the feeder train and $t^{\prime}$ the connecting train. Furthermore, the transfer time could be bounded by adding the following constraints to the timetabling model:

$$
L \leq \operatorname{rel}_{t^{\prime}, p^{\prime}}^{a u x}-\operatorname{res}_{t, p}^{a u x}+P \operatorname{relres}_{t, t^{\prime}, p}^{\mathrm{int}} \leq U
$$

where $t$ and $t^{\prime}$ are again the feeder and connecting train, $p$ and $p^{\prime}$ the platforms assigned in the

routing model, relres $\mathrm{int}_{t, p}^{\mathrm{int}}$ is an integer variable to account for the periodicity and $L$ and $U$ are appropriate bounds for the transfer time. Remark that we take here the difference of a release 
time and a reservation time, and not the difference of a reservation time and a release time as in constraints (19)-(22), such that the resrel ${ }^{\text {int }}$ variables cannot plainly be used here. That is why we introduced here the relres ${ }^{\text {int }}$ variables. It is easy to relate the resrel ${ }^{\text {int }}$ variables to the relres ${ }^{\text {int }}$ variables, but this is not necessary to build a correct model. Also easily the release variable, rel ${ }^{\text {aux }}$, and the reserve variable, res $^{\text {aux }}$, in these equations can be replaced by variables to indicate the arrival and departure times of the trains on the platform. Constraints must then be included that present the relation between these arrival and departure variables and the rel ${ }^{\text {aux }}$ and res ${ }^{\text {aux }}$ variables and the appropriate values for $L$ and $U$ must be slightly altered. Unfortunately, there is no data available on passenger transfers in Brussels, so we did not include these constraints in our model.

\subsection{Simulation}

The constructed timetable and routing plan are simulated with the simulation tool described in Dewilde et al. (2014). The same blocking time length calculations are considered in the timetabling model and the simulation and match with the data used by Dewilde et al. $(2013,2014)$. The simulation tool performs 10000 simulation runs. For each run, a delay for entering the network is drawn for about $50 \%$ of the trains from an exponential distribution with its average in the interval [0 min, $8 \mathrm{~min}$ ], based on historical data. The output of the simulation tool is specified in Section 5 .

\section{$5 \quad$ Results}

In this section, we discuss the performance of the developed routing model and timetabling model on the case study of Brussels. First, the results of the routing model and thereafter the timetabling and simulation results are presented.

\subsection{Routing results}

Table 6 shows the results of the routing model for different amounts of trains (with half of the trains going from Brussels North to Brussels South and the other half in opposite direction). The calculations are done with CPLEX 12.6 on an Intel Core i7-5600U CPU @ 2.60 GHz. We also added the values for the reference routing plan for 85 trains from Infrabel and from Dewilde et al. (2013) in the first two lines. In the first column is indicated if constraints (7) or (4) are used in the routing model. This is not applicable for the reference routing plan of Dewilde et al. (2013) and Infrabel. The second column contains the number of trains that is assigned to a route. In the third column, the time to construct the routing model is given. We see that the computation time that CPLEX needs to construct the routing model increases rapidly with the amount of trains, but is still acceptable for the 85 reference trains. The computation time to solve the routing model, in the fourth column, also increases with the amount of trains but remains (surprisingly) very short. The fifth column 
shows the maximum node usage. This maximum equals 16 for the case with 85 trains and can be explained by the line plan since 16 trains arrive via the same boundary point in the considered network. The sum of the squared node usages in the sixth column shows an improvement of $13.13 \%$ compared to the reference routing plan of Infrabel. The last line presents the results of the routing model with constraints (4) of Burggraeve et al. (2015) instead of our constraints (7). We see that the model construction time of both alternatives is about the same, but that the computation time to solve the model differs significantly. While our model solves in some seconds, the model with constraints (4) is not even solved up to optimality within 12 hours.

Table 6: Results from the routing model

\begin{tabular}{ccrrrrr} 
constraints & $\#$ trains & construct time & comp time & $\max g_{w}$ & $\sum_{w \in W} g_{w}^{2}$ & impro \\
\hline- & 85 & Infrabel & 16 & 27565 & - \\
- & 85 & Dewilde & 16 & 27410 & $-0.56 \%$ \\
\hline$(7)$ & 20 & $44 \mathrm{~s}$ & $8 \mathrm{~s}$ & 6 & 1820 & - \\
& 40 & $232 \mathrm{~s}$ & $14 \mathrm{~s}$ & 8 & 6246 & - \\
& 60 & $607 \mathrm{~s}$ & $17 \mathrm{~s}$ & 12 & 13397 & - \\
& 85 & $1403 \mathrm{~s}$ & $26 \mathrm{~s}$ & 16 & 23945 & $-13.13 \%$ \\
\hline$(4)$ & 85 & $1377 \mathrm{~s}$ & $>12 \mathrm{~h}$ & 16 & 23978 & $-13.01 \%$
\end{tabular}

\subsection{Timetabling and simulation results}

In Table 7, we show the robustness results of the solutions of the routing model and the timetabling model. We compare their performance with the results of a reference timetable and routing plan from Infrabel from 2010 and the best found routing plan and timetable for Brussels by Dewilde et al. (2013). For the optimal routing plan for 85 trains discussed above, we experimented with including different amount of supplements in the timetable: $0 \%, 25 \%, 41 \%, 50 \%$ of the dwell time is added as a supplement. The reference timetable of Dewilde et al. (2013) contains the same dwell and running times and the same (dwell and running time) supplements as the reference timetable from Infrabel, about 203 minutes. Note that without supplements a delay can never be absorbed. Including $41 \%$ of dwell time supplements gives us a total amount of supplements as close as possible to the timetable of Infrabel and Dewilde et al. (2013) (not exactly due to a time discretization of $0.1 \mathrm{~min}$ for these supplements). We calculated timetables 1-2-3-4 by using a compute node Xeon E5-2680v2, IB-QDR, 20 cores with 16 GB memory for three hours and for timetable 5 with 64 GB memory for 24 hours. Apart from the difference in computation time and available memory between timetables 1-2-3-4 on the one hand and timetable 5 on the other hand, all five timetables are constructed starting from the same routing plan, but differ in the amount of dwell time supplements. We see an improvement in passenger robustness (column passenger robustness) of $11.42 \%$ for timetable 3 (column impro) 
compared to the reference timetable of Infrabel. This is also better than the improvement of $9.43 \%$ of the timetable found by Dewilde et al. (2013). The column supplement reports the amount of dwell time supplements included in the timetables. The more dwell time supplement is added, the less trains leave the network with a delay (column delay out) and the smaller the best found minimum buffer time after three hours of optimization (column min buffer time). Note that these minimum buffer times are very short, but that the safety headways between the trains, defined by the signals, are respected and that these buffer times should be considered as additional. We see that the passenger robustness is very bad if no supplements are included in the timetable. The passenger robustness improves if more supplements are included, but deteriorates again if $50 \%$ dwell time supplements are included. If the passenger robustness does not improve anymore by adding more supplements, an equilibrium between supplements and buffer times is achieved. The column nominal travel time shows that our routing model constructed a routing plan with a shorter nominal travel time than that of the routing plan of Infrabel and Dewilde et al. (2013). This nominal travel time is the planned travel time without weights distinguishing between necessary running time or dwell time and supplements or transfer time. For timetable 5, we see that the best found minimum buffer time is significantly better and the number of trains which leave the network with a delay improves, while the passenger robustness deteriorates. This can be explained by the fact that the optimization models do not take passenger numbers into account while planning the trains. The impact of a delay on the passenger robustness increases proportionally with the number of passengers on the delayed train. The last column presents the number of deadlocks reported by the simulation module. This is the number of scenarios which are thrown away because two trains end up in each others next section. Actually, this is a drawback of the simulation module. It cannot predict or handle this situation since it only contains straightforward conflict resolution techniques. Anyway, for timetable 3 and 5 this number is comparable to that of the timetable found by Dewilde et al. (2013).

These results show that we can construct a routing plan and timetable from scratch which perform

Table 7: Simulation results

\begin{tabular}{|c|c|c|c|c|c|c|c|c|}
\hline timetable & $\begin{array}{l}\text { passenger } \\
\text { robustness } \\
\quad(\min )\end{array}$ & impro & $\begin{array}{l}\text { suppl } \\
\text { (min) }\end{array}$ & ment & $\begin{array}{c}\text { nominal } \\
\text { travel time } \\
\left(\cdot 10^{6} \mathrm{~min}\right)\end{array}$ & $\begin{array}{l}\text { minimum } \\
\text { buffer time } \\
\text { (s) }\end{array}$ & $\begin{array}{c}\text { delay } \\
\text { out } \\
(\%)\end{array}$ & deadlocks \\
\hline Infrabel & 2885049 & - & 203 & & 1.35 & - & 60.19 & 1687 \\
\hline Dewilde & 2612906 & -9.43 & 203 & & 1.35 & - & 49.17 & 5174 \\
\hline 1 & 3326365 & +15.30 & 0 & $(0 \%)$ & 1.34 & 5 & 80.81 & 47361 \\
\hline 2 & 2731927 & -5.31 & 118 & $(25 \%)$ & 1.34 & 4 & 59.65 & 27229 \\
\hline 3 & 2555668 & -11.42 & 206 & $(41 \%)$ & 1.34 & 1 & 42.99 & 6796 \\
\hline 4 & 2775147 & -3.81 & 226 & $(50 \%)$ & 1.34 & 0 & 46.01 & 49056 \\
\hline 5 & 2630333 & -8.83 & 206 & $(41 \%)$ & 1.34 & 17 & 41.83 & 4696 \\
\hline
\end{tabular}


at least as good as the reference routing plan and timetable from Infrabel and the best found routing plan and timetable for Brussels from literature constructed by Dewilde et al. (2013).

\section{Conclusion and future research}

In this paper we present a routing model and a timetabling model to construct a conflict-free and passenger robust routing plan and timetable from scratch for complex railway station areas. The line planning for the whole network is used as input. The resulting timetable for the bottleneck can serve as a starting point for the timetable of the whole network. The focus of these models is on optimally using the infrastructure in the bottleneck area and spreading the trains in time such that the passenger travel time in practice is optimized. We validate these models by constructing a conflict-free routing plan and timetable for Brussels' complex railway station area with the presented models. Furthermore, we compared the performance of this timetable and routing plan with a reference timetable and routing plan from the Belgian railway infrastructure manager Infrabel and with the best timetable from literature for this area constructed by Dewilde et al. (2013). The node usage is significantly decreased by $13 \%$. Moreover, simulation of the constructed routing plan and timetables show that our approach outperforms the passenger robustness of the reference routing plan and timetable from Infrabel with up to $11 \%$. This is even $2 \%$ better than the best routing plan and timetable from Dewilde et al. (2013) for this area.

A first idea for future research is to include the assignment of supplements into the timetabling model. With the current objective to maximize the buffer times, the model would generally not include running and dwell time supplements in order to keep the travel times as short as possible. Therefore, supplements will only be added to increase buffer times somewhere further in the network and not necessarily where they are useful in order to avoid the propagation of delays. A stochastic passenger travel time objective could weigh the advantages of both buffer times and dwell times in the construction of a timetable. However, an exact model with this objective that is solved in a reasonable amount of time for microscopic timetabling in complex railway stations is a big challenge. Including passenger numbers in the optimization models is another idea for future research. We foresee that this will further improve the passenger robustness.

\section{Acknowledgements}

This research was supported in part through computational resources provided by the KU Leuven high performance cluster. We thank the Belgian infrastructure manager Infrabel for their commitment during the research and for providing data. We also thank Gábor Maróti for the fruitful discussion on the CASPT2015 conference in Rotterdam about the linearization of the routing objective function. 


\section{References}

Andersson, E.V., Peterson, A., Törnquist Krasemann, J., Improved railway timetable robustness for reduced traffic delays - a MILP approach, In: Proceedings of the 6th International Seminar on Railway Operations Modelling and Analysis (RailTokyo2015), Tokyo, Japan (2015).

Bešinović, N., Goverde, R.M.P., Quaglietta, E., Roberti, R., An integrated micro-macro approach to robust railway timetabling, Transportation Research Part B, vol. 87, pp. 14-32 (2016).

Bešinović, N., Goverde, R.M.P., Quaglietta, E., Microscopic models and network transformations for automated railway traffic planning, Computer-Aided Civil and Infrastructure Engineering, vol. 32(2), pp. 89-106 (2017).

Burggraeve, S., Dewilde, T., Sels, P., Vansteenwegen, P., Improving passenger robustness by taking passenger numbers and recurring delays into account on the tactical level, In: Proceedings of the 6th International Conference on Railway Operations Modelling and Analysis (RailTokyo2015), pp. 1-15 (2015).

Burggraeve, S., Van Thielen, S., Vansteenwegen, P. (2015). Advanced methods for improving passenger robustness in railway systems, In: Proceedings of the Conference on Advanced Systems in Public Transport (CASPT2015), Rotterdam, Netherlands (2015).

Dewilde, T., Sels, P., Cattrysse, D., Vansteenwegen, P., Defining robustness of a railway timetable, In: Proceedings of 4th International Seminar on Railway Operations Modelling and Analysis (RailRome2011), Rome, Italy (2011).

Dewilde, T., Sels, P., Cattrysse, D., Vansteenwegen, P., Robust railway station planning: An interaction between routing, timetabling and platforming, Journal of Rail Transport Planning \& Management, vol. 3, pp. 68-77 (2013).

Dewilde, T., Sels, P., Cattrysse, D., Vansteenwegen, P., Improving the robustness in railway station areas, European Journal of Operational Research, vol. 235, pp. 276-286 (2014).

Cacchiani, V., Toth, P., Nominal and robust train timetabling problems, European Journal of Operational Research, vol. 219, pp. 727-737 (2012).

Caimi, G. C., Algorithmic decision support for train scheduling in a large and highly utilised railway network, Diss., Eidgenössische Technische Hochschule ETH Zürich, Nr. 18581 (2009).

Caimi, G., Chudak, F., Fuchsberger, M., Laumanns, M., Zenklusen, R., A new resource-constrained multicommodity flow model for conflict-free train routing and scheduling, Transportation science, vol. 45(2), pp. 212-227 (2011).

Fischetti, M., Salvagnin, D., Zanette, A., Fast approaches to improve the robustness of a railway timetable, Transport Science, vol. 43, pp. 321-335 (2009).

Goldratt, E.M., Cox, J., The Goal: A Process of Ongoing Improvement, North River Press (1986). Goverde, R.M.P., Bešinović, N., Binder, A., Cacchiani, V., Quaglietta, E., Roberti, R., Toth, P., A three-level framework for performance-based railway timetabling, Transportation Research Part 
C, vol. 67, pp. 62-83 (2016).

Kroon, L., Maroti, G., Helmrich, M., Vromans, M., Dekker, R., Stochastic improvement of cyclic railway timetables, Transportation Research Part B, vol. 42, pp. 553-570 (2008).

Kroon, L., Huisman, D., Abbink, E., Fioole, P., Fischetti, M., Maróti, G., Schrijver, A., Steenbeek, A., Ybema, R., The New Dutch Timetable: The OR Revolution, Interfaces, vol. 39(1), pp. 6-17, (2009).

Lamorgese, L., Mannino, C., Natvig, E., An exact micro-macro approach to cyclic and non-cyclic train timetabling, Omega, http://dx.doi.org/10.1016/j.omega.2016.11.004 (2016).

Liebchen, C., Möhring, R., The modeling power of the periodic event scheduling problem: Railway timetables - and beyond, Algorithmic methods for railway optimization, In: Lecture Notes in Computer Science, Ed. by F. Geraets, L. Kroon, A. Schöbel, D. Wagner, D. Zaroliagis, vol.4359, Springer Berlin Heidelberg, pp. 3-40 (2007).

Lusby, R.M., Larsen, J., Ehrgott, M., Ryan, D., Routing trains through railway junctions: A new set-packing approach, Transportation Science, vol. 45, pp. 228-245 (2011).

Pachl, J., Timetable design principles, In: Railway timetable \& traffic: Analysis, modelling and simulation, Ed.: Hansen, I. and Pachl, J., Eurailpress, Hamburg, pp. 9-42 (2008).

Schlechte, T., Borndörfer, R., Erol, B., Graffagnino, T., Swarat, E., Micro-macro transformation of railway networks, Journal of Rail Transport Planning \& Management, vol. 1(1), pp. 38-48 (2011).

Schmidt, M., Schöbel, A., Timetabling with passenger routing, OR Spectrum, vol. 37(1), pp. 75-97 (2015).

Sels, P., Vansteenwegen, P., Dewilde, T., Cattrysse, D., Waquet, B., Joubert, A., The train platforming problem: The infrastructure management company perspective, Transportation Research Part B: Methodological 61, pp. 55-72 (2014).

Sels, P., Dewilde, T., Cattrysse, D., Vansteenwegen, P., Reducing the passenger travel time in practice by the automated construction of a robust railway timetable, Transportation Research par B: Methodological 84, pp. 124-156 (2016a).

Sels, P., Large-Scale, Passenger Oriented, Cyclic Railway Timetabling and Station Platforming and Routing, PhD thesis, KU Leuven, Leuven, May 2016, pp. 4-10 (2016b).

Serafini, P., Ukovich, W., A Mathematical model for periodic scheduling problems, SIAM Journal on Discrete Mathematics, vol. 2, pp. 550-581 (1989).

Sun, Y., Cao, C., Wu, C., Multi-objective optimization of train routing problem combined with train scheduling on a high-speed railway network, Transportation Research Part C, vol. 44, pp. 1-20 (2014).

UIC, Code 406: Capacity (second ed.)International Union of Railways, Paris (2013).

Zwaneveld, P.J., Kroon, L.G., Romeijn, H.E., Salomon, M., Dauzère-Pérès,S., van Hoesel, S.P.M., Ambergen, H.W., Routing trains through railway stations: Model formulation and algorithms, 
Transportation Science, vol. 30, pp. 181-194 (1996).

Zwaneveld, P.J., Kroon, L.G., van Hoesel, S.P.M., Routing trains through a railway station based on a node packing model, European Journal of Operational Research, vol. 128, pp. 14-33 (2001). 